\title{
The morphology of perinecrotic tumor cell nuclei in glioblastomas shows a significant relationship with survival time
}

\author{
REINHOLD NAFE ${ }^{1}$, KEA FRANZ ${ }^{2}$, WOLFGANG SCHLOTE ${ }^{3}$ and BERTHOLD SCHNEIDER ${ }^{4}$ \\ ${ }^{1}$ Department of Neuroradiology, ${ }^{2}$ Clinics of Neurosurgery, ${ }^{3}$ Department of Neuropathology (Edinger-Institute), \\ Clinics of Johann Wolfgang Goethe-University, Schleusenweg 2-16, D-60528 Frankfurt am Main; \\ ${ }^{4}$ Department of Biometrics, Carl-Neubergstrasse 1, Medical School, D-30625 Hannover, Germany
}

Received March 2, 2006; Accepted May 17, 2006

\begin{abstract}
A deeper knowledge about histopathological criteria with a significant impact on the prognosis of patients with glioblastomas is worthwhile, since these patients may show a considerable difference in the time of survival. Investigation of the morphology of perinecrotic tumor cell nuclei is a promising approach, because the expression of specific molecules in these cells has been associated with a more aggressive behaviour of the tumors. In our series of patients with documented clinical course, 11 patients had a survival of at least 24 months and we compared this group with a group of 10 patients with maximum survival of 12 months. Digital microscopic image analysis was performed in paraffin sections from the primary surgical specimen. Three hundred perinecrotic tumor cell nuclei per case and 300 nuclei per case from tumor cells lying more distant from the tumor necroses ('distant zone' nuclei) were measured. The ratio for the numerical nuclear density between both types of nuclei was significantly larger in cases with short survival indicating a more pronounced nuclear density of perinecrotic nuclei in relation to the 'distant zone' nuclei in these tumors. In cases with long survival, on the contrary, perinecrotic tumor cells exhibited an only slightly larger numerical density compared with 'distant zone' nuclei. Mean values and standard deviations from parameters of nuclear shape (Fourier-amplitudes) had significantly smaller values in short-time survivors indicating a tendency towards a more circular nuclear shape with less pronounced intratumoral variation in that group. Based on the morphometric results, all cases could be correctly reclassified as short- or long-time survivors by means of cross-validated discriminant analysis. In conclusion, the results confirm significant morphological differences between tumors from patients with short and with
\end{abstract}

Correspondence to: Dr Reinhold Nafe, Department of Neuroradiology, Clinics of Johann Wolfgang Goethe-University, Schleusenweg 2-16, D-60528 Frankfurt, Germany

E-mail: r.nafe@em.uni-frankfurt.de

Key words: glioblastomas, prognosis, morphometry, tumor cell nuclei, necrosis long survival regarding morphology of both types of tumor cell nuclei. It seems likely, that histomorphometry of tumor cell nuclei could be a promising approach for the assessment of the individual prognosis of patients with glioblastomas.

\section{Introduction}

Although glioblastomas generally have a poor prognosis, some patients may show an unexpectedly long survival up to several years. Therefore, a deeper knowledge about prognostic factors is worthwhile, also with respect to therapeutic planning and the development of future therapeutic strategies. The need for the investigation of potential prognostic factors is further emphasized by the frequency and, thus, clinical importance of glioblastomas, which constitute the most frequent type of brain tumors together with meningiomas. Up to now, gross surgical resection, younger age of the patient, as well as local postoperative radiation are the best known prognostic factors being associated with a more favourable outcome $(1,2)$. In recent years, research on prognostic factors was mainly focussed on expression profiles and specific molecular biological alterations of the tumors $(3,4)$, whereas only few authors focussed on histomorphology of glioblastomas $(5,6)$. In a previous study, we showed that quantitative morphology of tumor cell nuclei in the region with the highest Ki67proliferation index is significantly related with survival time (7). Perinecrotic tumor cell nuclei constitute another challenge for the morphological investigation of glioblastomas, since these nuclei may show a distinct morphological variation from case to case and they often show a different morphology when compared with those tumor cell nuclei being located more distant from tumor necroses. A recent observation confirmed, that specific expression profiles of perinecrotic ('palisading') tumor cells are associated with poor clinical outcome (8). However, quantitative morphology of perinecrotic tumor cells as well as the prognostic significance of that morphology have not been previously investigated. The following study should contribute to the discussion of the following questions: i) What are the main quantitative morphological differences between perinecrotic tumor cell nuclei and those tumor cell nuclei located more distant from tumor necroses? ii) Are there significant differences between the tumors from a collective of patients with long survival (minimum: 24 months) and from a collective of patients with 
Table I. Data from 11 patients with a survival of at least 24 months and data from 10 patients with a maximum survival of 12 months.

\begin{tabular}{|c|c|c|c|c|c|c|c|}
\hline Patient & $\begin{array}{c}\text { Age } \\
\text { (years) }\end{array}$ & Sex & $\begin{array}{l}\text { Time of } \\
\text { survival } \\
\text { (months) }\end{array}$ & $\begin{array}{l}\text { Localization } \\
\text { of the tumor }\end{array}$ & $\begin{array}{c}\text { Histological signs } \\
\text { of a secondary } \\
\text { glioblastoma }\end{array}$ & $\begin{array}{l}\text { Amount of } \\
\text { band-like } \\
\text { necroses }\end{array}$ & $\begin{array}{l}\text { Amount of } \\
\text { large areas } \\
\text { of necroses }\end{array}$ \\
\hline 1 & 74 & M & 34 & $1-p$ & $*$ & 2 & 0 \\
\hline 2 & 49 & M & $31(>)$ & $1-f$ & $*$ & 1 & 2 \\
\hline 3 & 67 & M & 27 & $1-\mathrm{t}$ & - & 2 & 3 \\
\hline 4 & 72 & M & 26 & $r-t$ & - & 1 & 1 \\
\hline 5 & 39 & M & 46 & $1-f$ & $*$ & 1 & 1 \\
\hline 6 & 65 & M & $24(>)$ & $1-p$ & - & 1 & 3 \\
\hline 7 & 39 & M & $24(>)$ & $r-f$ & - & 2 & 1 \\
\hline 8 & 37 & $\mathrm{~F}$ & $31(>)$ & $r-f$ & - & 2 & 2 \\
\hline 9 & 71 & M & $38(>)$ & $r-f$ & - & 1 & 2 \\
\hline 10 & 55 & $\mathrm{~F}$ & $27(>)$ & $1-p$ & - & 2 & 0 \\
\hline 11 & 69 & M & $25(>)$ & $1-p$ & - & 1 & 2 \\
\hline 12 & 52 & $\mathrm{~F}$ & 12 & $r-t$ & - & 2 & 2 \\
\hline 13 & 60 & M & 12 & $r-t$ & $*$ & 1 & 3 \\
\hline 14 & 67 & $\mathrm{~F}$ & 7.5 & $r-f$ & - & 1 & 1 \\
\hline 15 & 52 & $\mathrm{M}$ & 7 & $r-t$ & - & 2 & 1 \\
\hline 16 & 77 & $\mathrm{M}$ & 6 & $r-t$ & - & 2 & 3 \\
\hline 17 & 58 & M & 9.5 & $r-f$ & - & 1 & 1 \\
\hline 18 & 65 & $\mathrm{M}$ & 5.5 & $r-f$ & - & 2 & 3 \\
\hline 19 & 48 & $\mathrm{M}$ & 10 & $1-f$ & - & 2 & 1 \\
\hline 20 & 72 & $\mathrm{~F}$ & 2 & $r-t$ & - & 1 & 0 \\
\hline 21 & 68 & $\mathrm{M}$ & 4.5 & $1-\mathrm{t}$ & - & 1 & 0 \\
\hline
\end{tabular}

$>$, patients still alive after the observation period, number of months for these patients indicates the minimum time of survival. Localization: 1, left; $r$, right; f, frontal; $p$, parietal; t, temporal. Semi-quantitative score for the amount of tumor necroses: 0 , none; 1 , few; 2 , distinct but not extensive; 3 , extensive.

short survival (maximum: 12 months) regarding the morphology of both types of tumor cell nuclei? iii) Do the results provide evidence that morphology of perinecrotic tumor cell nuclei (in relation to the morphology of those tumor cell nuclei more distant from necroses) can be regarded as an important morphological aspect for the assessment of the prognosis of patients with glioblastomas?

\section{Materials and methods}

Patients and tumor specimens. As a group of patients with considerably long survival, we investigated 11 glioblastomas from 11 patients surviving for at least 24 months. As a second group, we investigated 10 glioblastomas from 10 patients surviving for a maximum of 12 months (Table I). All patients under-went open surgery in the Department of Neurosurgery, Johann Wolfgang Goethe-University in Frankfurt am Main, Germany. Surgical specimen were embedded in paraffin after fixation in buffered formalin (4\%) and were typed and graded according to the newest edition of the WHO-classification of CNS-tumors (9). Stringent inclusion criteria for this study were formulated as follows: clear documentation of the clinical course of the patients; confirmation of the histological diagnosis of a glioblastoma WHO-grade IV by two neuropathologists; no residual tumor in postoperative MRI; only patients who died of the tumor disease were considered and not those who died of other reasons; a tumor specimen from the first surgical resection only were taken (no recurrent tumors); sufficient histological quality; areas of primary tumor necroses had to be present; tumor-localization had to be superficial (cortical) and thus no cases with deepseated glioblastomas from the basal ganglia or tumors from the brain-stem were investigated. All patients received a standardized post-operative local radiation with 60 Gy. All tumors had undergone routine histopathological examination and were reevaluated for this study by two neuropathologists. The amount of band-like necroses and of large areas of necroses in the surgical specimen was scored as follows: 0, none; 1 , few; 2, distinct but not extensive; 3 , extensive. It was also investigated, whether the tumor was likely to be a primary glioblastoma or if there was evidence for a secondary genesis out of a glioma with lower tumor grade. The latter was found to be likely in four cases showing large tumor areas of less malignancy resembling gliomas with 
WHO-tumor grades II or III (Table I). Morphometric investigations were performed in $4 \mu \mathrm{m}$ paraffin-sections stained with an antibody against the Ki67-antigen (Dako, Hamburg, Germany) and hematoxylin as counter-staining.

Morphometry. Measurements were performed by means of a digital image analysis system (KS400, Zeiss, Oberkochen, Germany). This system consists of a black/white-video camera mounted on a microscope and connected to a PC. Tumor cell nuclei were traced manually on the computer screen using the computer mouse by interactive control within the microscope (objective $\mathrm{x} 40$; numeric aperture: 0.75 ; tube factor: 1.25). Each nucleus was given the category 'Ki67-positive' or 'Ki67-negative' by the observer. Scoring for the amount of necroses showed the presence of band-like necroses in each tumor case (Table I). Furthermore, morphology of perinecrotic tumor cell nuclei around these necroses appeared to be different when compared with tumor cell nuclei being located more distant from necroses, especially with regard to a larger numerical nuclear density of perinecrotic tumor cell nuclei. Therefore, morphometric investigation was performed separately for both types of nuclei: in each case, 300 randomly selected perinecrotic tumor cell nuclei located around band-like necroses were measured, as well as 300 randomly selected tumor cell nuclei being located more distant from the necroses. The latter nuclei will be termed 'distant zone' nuclei in the following, which stands for 'distant from necroses'. In each case, the approximate linear thickness of the layer of perinecrotic tumor cell nuclei was measured using the digital image analysis system, i.e. the thickness of the layer of tumor cells adjacent to necroses, which show a rather homogeneous cytomorphology and numerical nuclear density clearly contrasting with tumor cells being located more distant from the necroses. The mean thickness of this layer from all cases was $86 \pm 14 \mu \mathrm{m}$ (mean \pm standard deviation). Perinecrotic tumor cell nuclei were measured within this layer. Those tumor cell nuclei lying less than 3 layers of nuclei away from the border of the necrosis or less than 3 layers of nuclei away from the outer border of this layer were not measured in order to avoid measurement of nuclei from necrotic tumor cells or even from tumor cells not clearly separated from the more distant tumor cells. On the other hand, the 'distant zone' tumor cell nuclei were measured outside the layer of the perinecrotic tumor cells up to a radial distance of maximal $300 \mu \mathrm{m}$ from the necroses. Again, those nuclei lying less than 3 layers of nuclei away from the border between perinecrotic and 'distant zone' nuclei were not measured in order to avoid a mix up of perinecrotic and 'distant zone' nuclei. For each tumor cell nucleus, the digital image analysis system calculated several parameters of nuclear size, nuclear shape and internuclear distances (Table II). For nuclear shape analysis, well known shape factors were used such as the roundness factor, calculated form area and perimeter of the nucleus, as well as the ellipse shape factor, calculated as the ratio between the smallest and the largest elliptic axe (10). The shape factors have the value 1 for a circle and smaller values with increasing irregularity of the nuclear shape. Also, Fourier-amplitudes were determined, which have larger values the more the shape deviates from a regular circle. This method has been described in detail elsewhere. Fig. 1 shows three examples for the results of
Fourier-analysis (Fig. 1). All parameters of nuclear shape were calculated invariate to nuclear size and rotation. Additionally, the numerical nuclear density (number of nuclei per area) was determined for perinecrotic and for 'distant zone' tumor cell nuclei in quadratic reference fields as described previously (11). For each of these parameters and for each tumor case, mean and standard deviation were calculated. These data then served as single values characterizing one tumor case in statistical analysis.

Statistical analysis. The software 'SPSS' (SPSS, Chicago, USA) was used for statistical analysis. In a first step, the t-test for paired samples was used for performing an intra-tumoral comparison between perinecrotic and 'distant zone' tumor cell nuclei. This test was performed separately for the group of patients with long survival (group 1: patients 1-11) and for the group of patients with short survival (group 2: patients 12-21; Table I, Fig. 2). In a second step, a pairwise comparison between both groups of patients was performed using multivariate analysis of variance. This analysis was performed regarding morphometric data from both types of tumor cell nuclei (perinecrotic and 'distant zone' nuclei) separately, as well as the patient's age and the scores for the amount of necrosis (Tables I and II, Figs. 3-5). In order to test for the degree of statistical separation between both groups of patients based on the morphometric results, a linear discriminant analysis was performed cross-validated (Fig. 6). In contrast to original discriminant analysis, cross-validated discriminant analysis provided the automatic performance of a 'leave-one-out-classification' and is therefore a good estimate for the prognostic significance of the present set of morphometric data. The whole statistical analysis has been performed as described in a review on statistical methods in histomorphometry (12).

\section{Results}

Intratumoral comparison of perinecrotic and 'distant zone' tumor cell nuclei showed a significant difference regarding the numerical nuclear density, which has been larger for perinecrotic tumor cell nuclei. This difference was significant for cases with long survival (group 1, n=11) and for cases with short survival (group 2, n=10) (Table I, Fig. 2). However, this difference was more pronounced in cases with short survival indicating a distinct numerical nuclear density of perinecrotic tumor cell nuclei when compared with 'distant zone' nuclei (Fig. 2). No significant differences between perinecrotic and 'distant zone' nuclei were found regarding parameters of nuclear size, nuclear shape or the Ki67-proliferation index.

The comparison between both groups of patients showed a slightly larger mean age for the group of patients with short survival (62 vs. 58 years), but this difference was not significant. Also the mean scores for the amount of band-like necroses (1.45 vs. 1.50) and for the amount of large areas of necroses (1.54 vs. 1.50) was not significantly different between the groups of patients. When compared with the group of patients with long survival, a larger ratio for the numerical nuclear density between perinecrotic and 'distant zone' tumor cell nuclei has been confirmed for the group of cases with short survival (Table II). This reflects the result of the intratumoral 
Table II. Morphometric data (mean values \pm standard deviation) of the tumor cell nuclei for cases with long survival $(\mathrm{n}=11)$ and for cases with short survival $(\mathrm{n}=10)$; Level of significance (multivariate analysis of variance).

Morphometric parameter

Cases with long survival

Cases with short survival

Significance

$(n=11)$

$(\mathrm{n}=10)$

Mean nuclear area $\left(\mu \mathrm{m}^{2}\right)$ -

perinecrotic nuclei:

$43.11 \pm 7.46$

$39.72 \pm 11.74$

'distant zone' nuclei:

$45.28 \pm 7.88$

$40.65 \pm 6.64$

Mean nuclear diameter $(\mu \mathrm{m})$ -

perinecrotic nuclei:

$9.73 \pm 0.70$

$9.31 \pm 1.41$

'distant zone' nuclei:

$9.97 \pm 0.76$

$9.51 \pm 0.57$

Mean roundness factor -

perinecrotic nuclei:

$0.833 \pm 0.019$

$0.846 \pm 0.003$

'distant zone' nuclei:

$0.835 \pm 0.018$

$0.844 \pm 0.017$

Mean ellipse shape factor -

perinecrotic nuclei:

$0.637 \pm 0.003$

$0.638 \pm 0.004$

'distant zone' nuclei:

$0.634 \pm 0.002$

$0.629 \pm 0.003$

Ki67-proliferation index (\%) -

perinecrotic nuclei:

$7.80 \pm 10.12$

$2.88 \pm 4.03$

$8.13 \pm 10.70$

$4.97 \pm 7.82$

Numerical nuclear density

(number/0.01 mm²) -

perinecrotic nuclei:

$47.36 \pm 20.51$

$52.70 \pm 14.69$

'distant zone' nuclei:

$41.94 \pm 19.34$

$37.76 \pm 8.93$

Distance between two

neighbouring nuclei $(\mu \mathrm{m})$ -

perinecrotic nuclei:

$9.85 \pm 1.75$

$8.89 \pm 1.75$

'distant zone' nuclei:

$9.85 \pm 1.42$

$10.19 \pm 2.06$

Mean distance between a nucleus

and the 8 nearest nuclei $(\mu \mathrm{m})$ -

perinecrotic nuclei:

$19.64 \pm 3.94$

$17.55 \pm 4.11$

'distant zone' nuclei:

$19.61 \pm 3.16$

$21.51 \pm 5.62$

Thickness of the perinecrotic tumor cell layer $(\mu \mathrm{m})$ :

$81.82 \pm 13.90$

$90.26 \pm 13.97$

Numerical nuclear density -

Ratio between perinecrotic

and 'distant zone' nuclei:

$1.139 \pm 0.059$

$1.395 \pm 0.198$

Distance between two

neighbouring nuclei -

Ratio between perinecrotic

and 'distant zone' nuclei:

$0.998 \pm 0.082$

$0.878 \pm 0.096$

**

Mean distance between a nucleus

and the 8 nearest nuclei -

Ratio between perinecrotic

and 'distant zone' nuclei:

$0.999 \pm 0.108$

$0.826 \pm 0.113$

**, $\mathrm{p}<0.01 ;-, \mathrm{p}>0.05$ (not significant). In contrast to Fourier-analysis of nuclear shapes (Fig. 3), no significant differences were observed regarding parameters of nuclear size, roundness and ellipse shape factor, proliferation index, numerical nuclear density, distances between the tumor cell nuclei, as well as the thickness of the perinecrotic tumor cell layer. In cases with short survival, however, the ratio between perinecrotic and 'distant zone' nuclei for the numerical nuclear density was significantly larger and the ratios for internuclear distances were significantly smaller. 

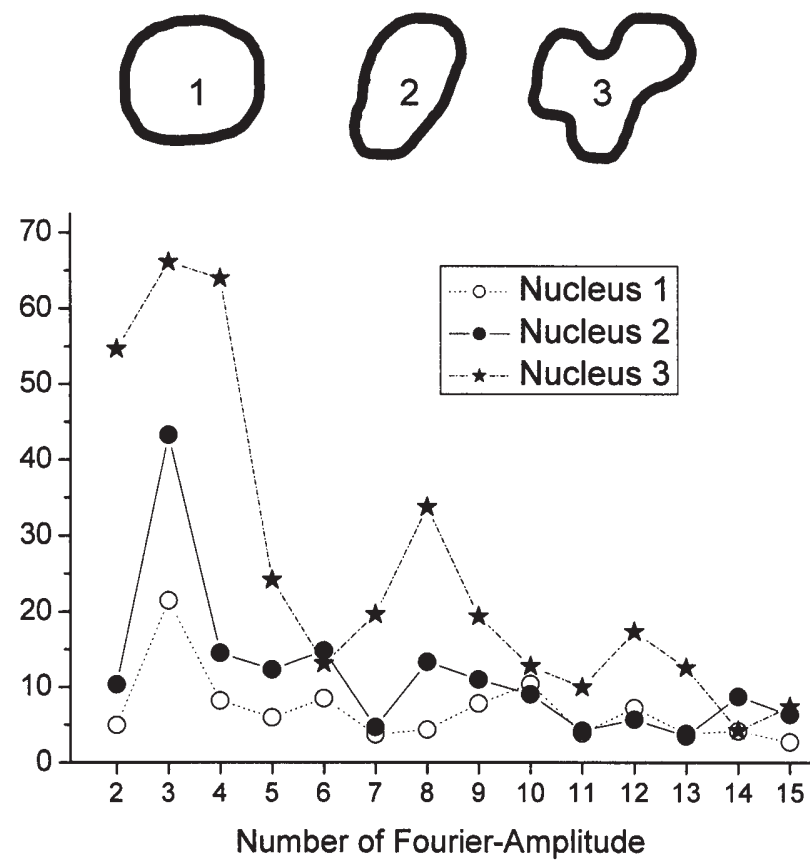

Figure 1. Fourier-analysis of nuclear shapes: calculation of Fourier-amplitudes nos. 2-15 (size-invariate and dimensionless) for three tumor cell nuclei. These amplitudes are good measures for the degree of shape irregularities of a tumor cell nucleus. Nucleus 1 has a nearly circular shape with only slight irregularities, Fourier-analysis shows low values for nearly all Fourieramplitudes; nucleus 2 has an elongated shape with larger values for Fourieramplitudes nos. 2-6; nucleus 3 shows distinct irregularities and thus larger values for most Fourier-amplitudes.

\section{Numerical density}

[number $/ 0.01 \mathrm{~mm}^{2}$ ]

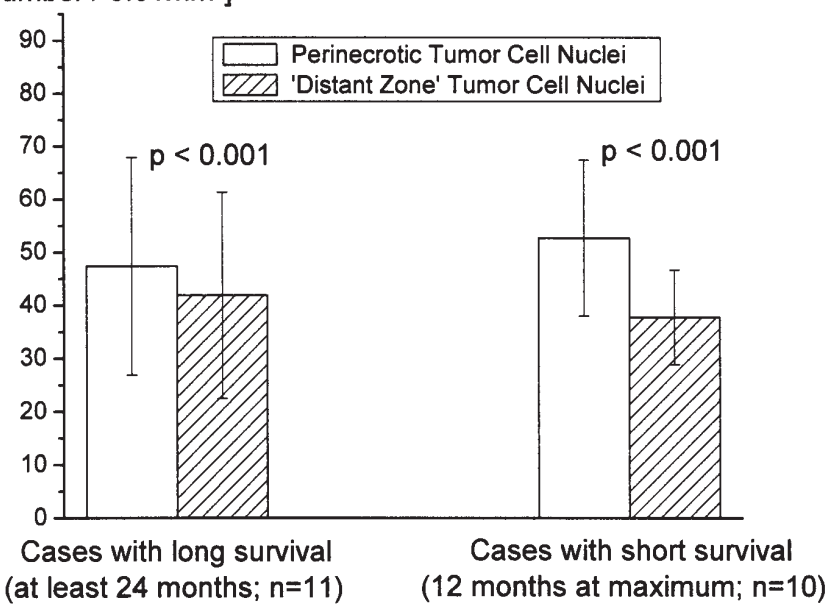

Figure 2. Intratumoral comparison between perinecrotic and 'distant zone' nuclei: a significantly larger numerical density of tumor cell nuclei can be shown for the perinecrotic tumor cell layer compared with the 'distant zone' nuclei (t-test for paired samples). This significant difference has been confirmed when analyzing cases with long survival and also when analyzing cases with short survival. However, the difference between perinecrotic and 'distant zone' nuclei is more pronounced in cases with short survival

comparison mentioned above, which showed a more pronounced difference between perinecrotic and 'distant zone' tumor cell nuclei in this group (Fig. 2). The ratios between perinecrotic and 'distant zone' nuclei for the mean nuclear distances were significantly smaller in this group indicating a
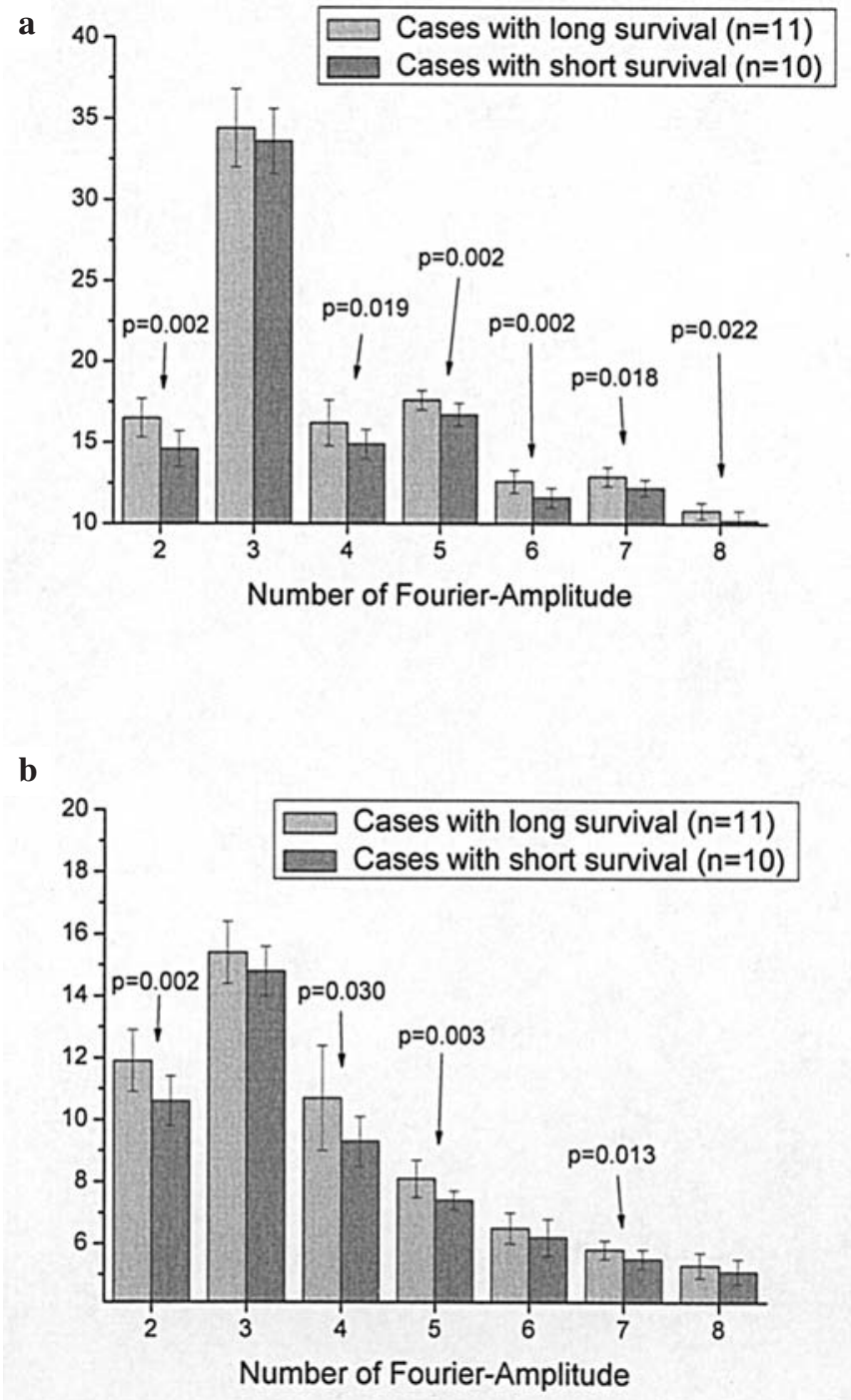

Figure 3. Comparison between cases with long survival $(n=11)$ and short survival $(n=10)$ : $(a)$, 'Distant zone' nuclei show lower mean values of several Fourier-amplitudes (size-invariate and dimensionless) in cases with short survival indicating a significantly more regular and circular nuclear shape in these cases; similar results for perinecrotic tumor cell nuclei showing lower mean values of Fourier-amplitudes nos. 2, 4, 5, 6 and 8 in cases with short survival; (b), Perinecrotic tumor cell nuclei show lower values for the standard deviations of several Fourier-amplitudes indicating a less pronounced intratumoral variation of nuclear shapes in cases with short survival; 'distant zone' nuclei show lower values for the standard deviations of Fourier-amplitudes nos. 5 and 7 in cases with short survival.

shorter internuclear distance for perinecrotic nuclei in relation to the 'distant zone' nuclei (Table II). No significant differences between the groups of patients were found for parameters of nuclear size, shape factors and Ki67-proliferation index. The mean thickness of the layer of perinecrotic tumor cells was larger in the group with short survival, but this difference was not significant (Table II). In contrast, Fourier-analysis showed lower values for mean values and also for standard deviations of several Fourier-amplitudes in the group with short survival (Fig. 3). These significant differences have been found for perinecrotic nuclei and also for 'distant zone' nuclei. This result indicates a tendency towards more regular (circular) nuclear shapes with a less pronounced intratumoral variation of nuclear shapes in patients with short survival. During 

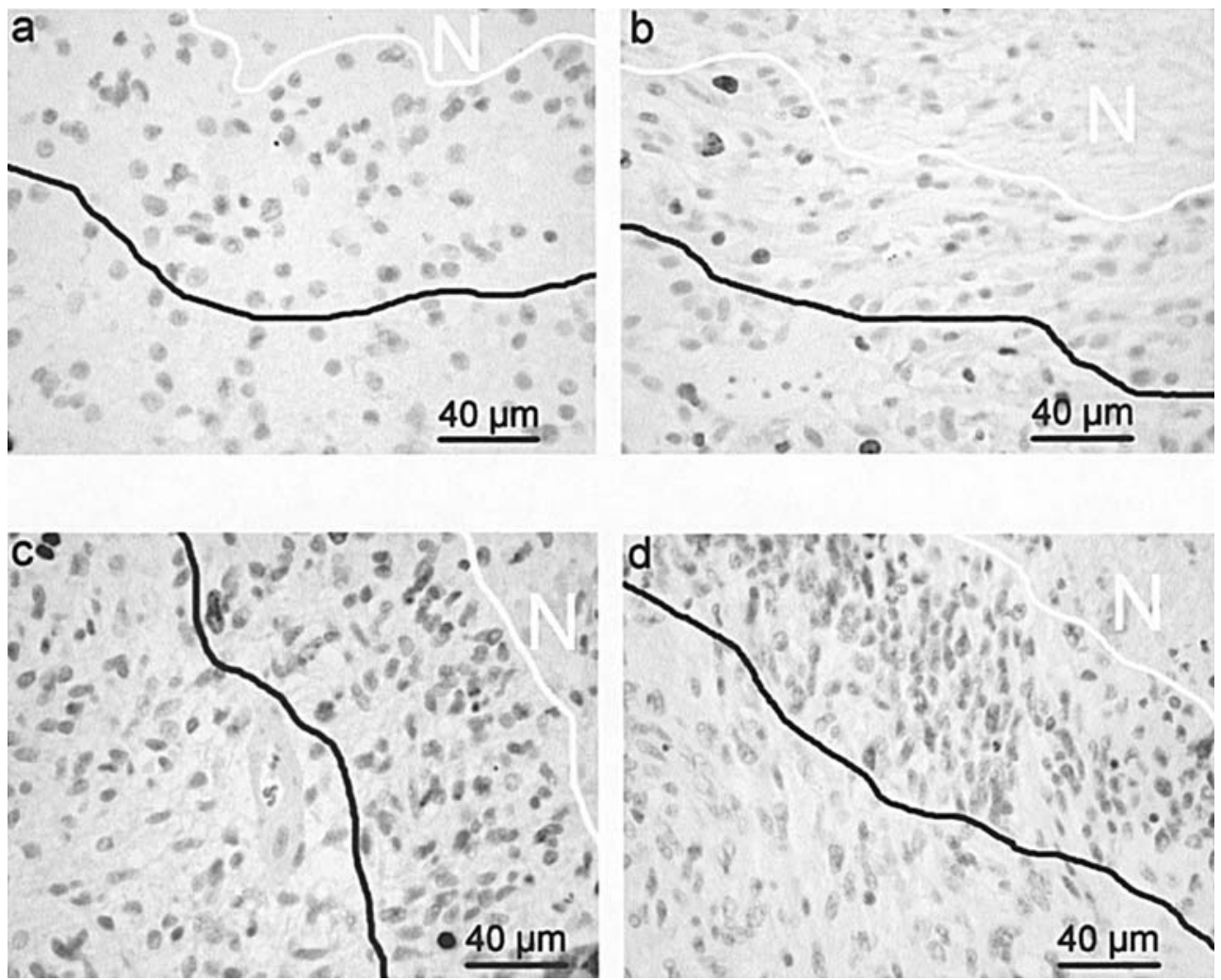

Figure 4. Histological images from patient 8 (a) and patient 10 (b) with long survival and from patient 13 (c) and patient 20 (d) with shorter survival (Table I): the white lines indicate the approximate border between tumor necrosis ('N') and the perinecrotic tumor cell layer, the black lines indicate the approximate border between the perinecrotic and the 'distant zone' tumor cells; (a, b), In cases with long survival, the perinecrotic tumor cell layer only shows a slightly larger numerical nuclear density compared with the nuclear density of the 'distant zone'; (c, d), In cases with short survival, the perinecrotic tumor cell layer shows a considerably larger numerical nuclear density compared with the nuclear density of the 'distant zone'.

microscopic re-evaluation of the cases after the morphometric investigations, the pronounced difference between the numerical nuclear density of perinecrotic and 'distant zone' nuclei could be confirmed for cases with short survival (Fig. 4), as well as the more circular and regular nuclear shape of perinecrotic and even 'distant zone' tumor cell nuclei, as being confirmed by the results of Fourier-analysis (Figs. 3 and 5).

Cross-validated linear discriminant analysis with the time of survival as grouping variable (group 1: long survival for at least 24 months; group 2: short survival up to 12 months) confirmed a $100 \%$ correct reclassification of all cases based on the morphometric results. Large absolute values for the discriminant scores showed a clear statistical separation of the groups of patients (Fig. 6). Of note, a 100\% correct reclassification was also achieved, when only parameters of nuclear shapes (Fourier-analysis) were considered. When just considering parameters of numerical nuclear density in the analysis, only $85.7 \%$ of the cases (18/21) were correctly reclassified, indicating a certain degree of overlap between the groups of patients regarding parameters of numerical nuclear density. The whole statistical analysis performed so far was repeated for all cases with the exception of the four cases showing histological signs of a secondary genesis of the glioblastoma (Table I). This analysis led to the same results as the first analysis: the group of patients with short survival showed a significantly larger ratio for the numerical nuclear density and smaller ratios for the internuclear distances (ratios between perinecrotic and 'distant zone' nuclei), smaller values for means and standard deviations of several Fourieramplitudes, as well as a $100 \%$ correct reclassification of all cases by means of cross-validated linear discriminant analysis.

\section{Discussion}

Glioblastomas still have a generally worse prognosis with less than $2 \%$ of the patients surviving at least 3 years irrespective of postoperative radiotherapy (13). From our own series of patients with glioblastomas, 11 patients had a survival of at least 24 months. Therefore, it was necessary to perform a pairwise comparison between these 11 tumors and tumors from patients with short survival regarding differences in histomorphology. Such a pairwise comparison has been performed by other authors reporting significantly fewer mitoses, a lower Ki67-proliferation index, and a higher frequency of tumor cells with $\mathrm{p} 53$ and mdm2-expression in patients with long-term survival $(6,13)$. A significant association with increased survival time has been confirmed for the expression of other proteins such as interleukin-1ß (14), while there are still contradictory reports about the influence of the expression of other proteins on survival time $(3,4,15)$. From histopathological criteria, only the amount of necroses has been associated with survival time $(5,16)$. However, radiological data have shown that a significantly longer survival can only be confirmed when necroses exhibited less than $35 \%$ of the tumor tissue. When necrosis involved more than $35 \%$ of the mass, 

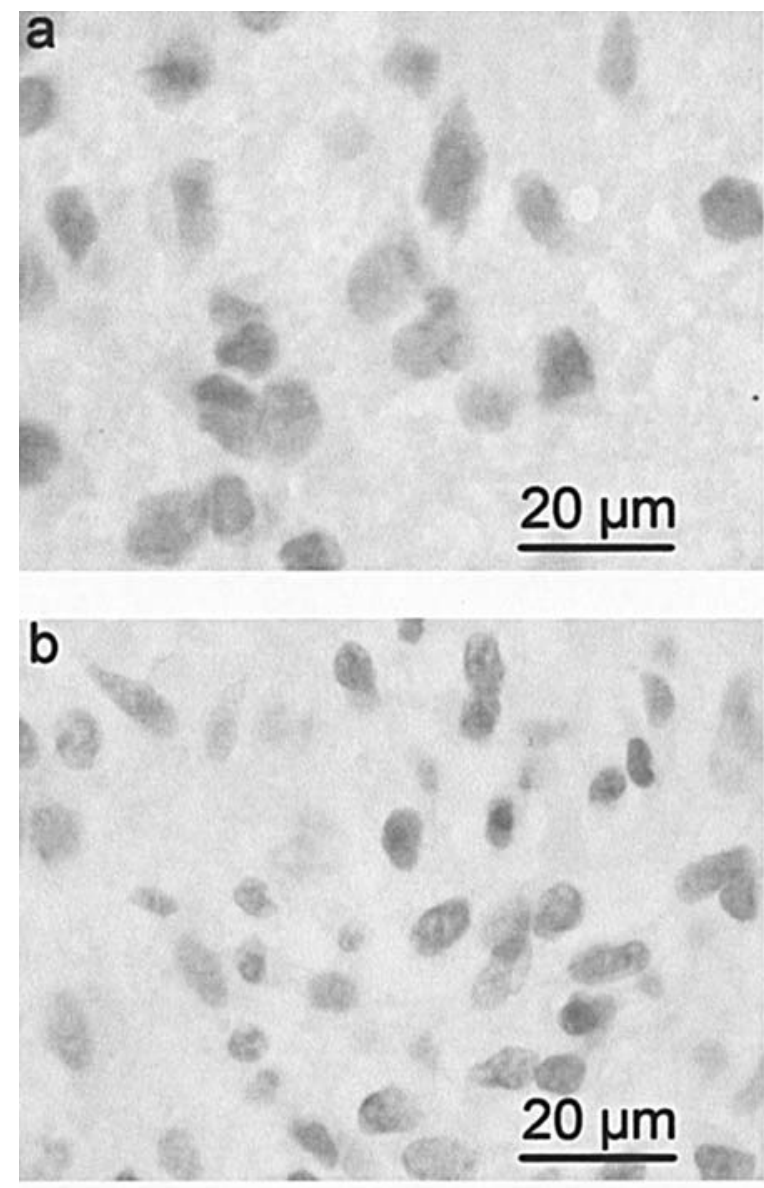

\section{C}

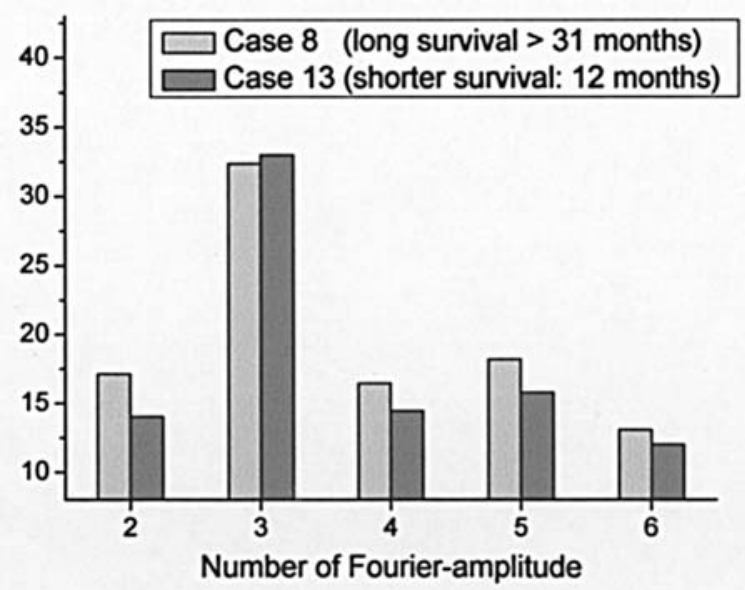

Figure 5. Tumor cell nuclei from the 'distant zone': nuclear shapes in case no. 8 (a) with long survival and in case no. 13 (b) with a shorter survival; significantly smaller values for several Fourier-amplitudes were found in cases with shorter survival indicating a tendency towards a more round and regular shape. This difference in nuclear shape is difficult to ascertain by visual examination alone; Fourier-analysis, however, clearly shows smaller values for several Fourier-amplitudes in the case with shorter survival (c).

no further correlation could be shown between survival time and the extent of necroses (17). This result is supported by our own histopathological study showing no significant difference between both groups of patients regarding the necrosis-scores (Table I). Especially, there were patients with long survival
- Patients with long survival $(n=11)$

- Patients with short survival $(n=10)$

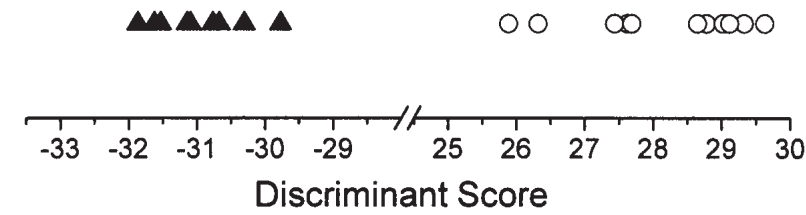

Figure 6. Linear discriminant analysis (cross-validated): $100 \%$ correct reclassification of all cases from group 1 (long survival of at least 24 months; $\mathrm{n}=11$ ) and from group 2 (short survival of a maximum of 12 months; $\mathrm{n}=10$ ). For each of the 21 cases, discriminant analysis calculated a discriminant score; the high absolute values for the discriminant scores indicate a distinct separation of both groups of patients.

but a distinct amount of tumor necroses (Table I, patients no. 3, 6 and 8). Therefore, the prognostic significance of the amount of tumor necroses in glioblastomas should be interpreted with caution in clinical practice and a large extent of necroses should not automatically be interpreted in terms of a worse prognosis. Taken together, these data support the need for investigating the prognostic significance of other morphological criteria, which could support prognostic assessment of individual patients in clinical practice.

A significant correlation between survival time and morphology of tumor cell nuclei has been confirmed in the region with the highest Ki67-proliferation index. The Ki67proliferation index itself, however, showed no significant association with survival time (7), which was also the fact in the present study on perinecrotic tumor cell nuclei and tumor cell nuclei more distant from necroses (Table II, Figs. 3-6). In the present study, typical band-like necroses with 'palisading' perinecrotic tumor cell nuclei were present in all cases irrespective of the length of survival time. As a striking difference between patients with long and with short survival, the ratio between the numerical nuclear density of these perinecrotic nuclei and the 'distant zone' nuclei was significantly larger in tumors from patients with short survival. Additionally, the shape of both types of nuclei tended to be more circular and regular in this group of patients (Figs. 3-5). Although palisading tumor cell nuclei around necroses occur frequently in glioblastomas, only few studies have focussed on that histological feature so far. Expression analysis found that nuclear 'palisades' around necroses represent a response to local hypoxic stress and show an expression profile which is distinct from those tumor cells more distant from tumor necroses $(18,19)$. It is postulated, that palisading tumor cells represent a clonal selection of cells with diminished apoptotic potential, that could underlie a more aggressive behaviour of the individual tumor $(8,16,20)$. Additionally, in tumors from patients with short survival, an elevated expression of several proteins has been found such as fucosyltransferase, which is involved in Notch signalling pathways, as well as phosphatidylserine factor, which is involved in clearance of apoptotic cells $(8,21)$. Our own results complement these observations by showing also morphological differences between different prognostic groups, independent of the differences regarding molecular biological alterations or expression profiles. Of note, our 
collective of patients is homogeneous with respect to localization (superficial cortical tumors) and age (no patients $<20$ years). This is an important point, since glioblastomas in childhood may show a more favourable clinical behaviour (22) and tumors from other sites such as deep-seated tumors may exhibit a different expression profile (23). Due to the standard postoperative treatment (local radiation with $60 \mathrm{~Gy}$ ), even effects of different treatment modalities on survival time could be excluded. Finally, a bias of the results due to differences regarding the mode of tumorigenesis (primary or secondary glioblastoma) could be excluded, since the statistical results from the entire database could be confirmed after exclusion of the four cases with evidence for a secondary glioblastoma.

The morphometric method implemented in this study shows a good reproducibility, as being tested in several tumor types including glioblastomas (24). It is important to perform a detailed morphometric analysis using a detailed set of morphometric parameters in order to test, which morphological criteria can contribute to the differentiation between prognostically different groups. A good example for this necessity is the clear difference in nuclear shape between both groups of patients as demonstrated by means of Fourieranalysis, although the other shape factors failed to show significant differences (Table II, Fig. 3). Therefore, testing for a large set of morphometric parameters was justified even for our set of patients, as previously indicated in a review on statistical methods in histomorphometry (12). Taken together, our results confirm that the morphology of perinecrotic tumor cell nuclei and those nuclei lying more distant from necroses show significant differences between patients with long and with short survival, especially with regard to numerical nuclear density and nuclear shape. The $100 \%$ correct statistical separation of both groups of patients supports the result of a previous study (7), that nuclear morphology must be considered a strong indicator of the prognosis of patients with glioblastomas. Histomorphometry is a valuable tool in order to provide a reproducible assessment of these morphological criteria.

\section{References}

1. Mineo JF, Quintin-Roue I, Lucas B, Buburusan V and Besson G: Glioblastomas: clinical study and search for prognostic factors. Neurochirurgie 48: 500-509, 2002.

2. Paszat L, Laperriere N, Groome P, Schulze K, Mackillop W and Holowaty E: A population based study of glioblastoma multiforme. Int J Radiat Oncol Biol Phys 51: 100-107, 2001.

3. Schmidt MC, Antweiler S, Urban N, et al: Impact of genotype and morphology on the prognosis of glioblastoma. J Neuropathol Exp Neurol 61: 321-328, 2002.

4. Zagzag D, Blanco C, Friedlander DR, Miller DC and Newcomb EW: Expression of p27KIP1 in human gliomas: relationship between tumor grade, proliferation index and patient survival. Hum Pathol 34: 48-53, 2003.
5. Burger PC and Green SB: Patient age, histologic features and length of survival in patients with glioblastoma multiforme. Cancer 59: 1617-1625, 1987.

6. Burton EC, Lamborn KR, Forsyth P, et al: Aberrant p53, mdm2 and proliferation differ in glioblastomas from long-term compared with typical survivors. Clin Cancer Res 8: 180-187, 2002.

7. Nafe R, Franz K, Schlote W and Schneider B: Morphology of tumor cell nuclei is significantly related with survival time of patients with glioblastomas. Clin Cancer Res 11: 2141-2148, 2005.

8. Dong S, Nutt CL, Betensky RA, et al: Histology-based expression profiling yields novel prognostic markers in human glioblastoma. J Neuropathol Exp Neurol 64: 948-955, 2005.

9. Kleihues P, Burger PC, Collins VP, Newcomb EW, Ohgaki H and Cavenee WK: Glioblastoma. In: Pathology and Genetics of Tumours of the Nervous System. Kleihues P and Cavenee WK (eds). International Agency for Research on Cancer, IARC-Press, Lyon, pp29-39, 2000.

10. Nafe R, Glienke W, Schlote W and Schneider B: EGFR geneamplification in glioblastomas - is there a relationship with morphology of tumor cell nuclei and proliferative activity? Analyt Quant Cytol Histol 23: 135-143, 2001.

11. Nafe R, Herminghaus S, Raab P, et al: Correlation between preoperative magnetic resonance spectroscopic data on high grade gliomas and morphology of Ki-67-positive tumor cell nuclei. Analyt Quant Cytol Histol 25: 131-138, 2003.

12. Nafe R and Schneider B: Data analysis for comparative histometry in pathology - I. classification procedures and other statistical methods. E J Pathol 6: 002-03.txt, 2000.

13. Scott JN, Rewcastle NB, Brasher PMA, et al: Which glioblastoma multiforme patient will become a long-term survivor? A population-based study. Ann Neurol 46: 183-188, 1999.

14. Cuny E, Loiseau H, Penchet G, et al: Association of elevated glial expression of interleukin-1 $\beta$ with improved survival in patients with glioblastomas multiforme. J Neurosurg 96: 294-301, 2002.

15. Rich JN, Hans C, Jones B, et al: Gene expression profiling and genetic markers in glioblastoma survival. Cancer Res 65: 4051-4058, 2005.

16. Raza SM, Lang FF, Aggarwal BB, et al: Necrosis and glioblastoma: a friend or a Foe? A review and hypothesis. Neurosurgery 51: 2-13, 2002.

17. Pierallini A, Bonamini M, Pantano P, et al: Radiological assessment of necrosis in glioblastoma: variability and prognostic value. Neuroradiology 40: 150-153, 1998.

18. Brat DJ and van Meir EG: Vaso-occlusive and prothrombotic mechanisms associated with tumor hypoxia, necrosis and accelerated growth in glioblastoma. Lab Invest 84: 397-405, 2004.

19. Brat DJ, Castellano-Sanchez AA, Hunter SB, et al: Pseudopalisades in glioblastoma are hypoxic, express extracellular matrix proteases, and are formed by an actively migrating cell population. Cancer Res 64: 920-927, 2004.

20. Graeber TG, Osmanian C, Jacks T, et al: Hypoxia-mediated selection of cells with diminished apoptotic potential in solid tumours. Nature 379: 88-91, 1996.

21. Fadok VA, Bratton DL, Rose DM, et al: A receptor for phosphatidylserine specific clearance of apoptotic cells. Nature 405: 85-90, 2000.

22. Cervoni L, Celli $P$ and Salvati M: Long-term survival in a patient with supratentorial glioblastoma: clinical considerations. Ital J Neurol Sci 19: 221-224, 1998.

23. Hayashi Y, Yamashita J and Watanabe T: Molecular genetic analysis of deep-seated glioblastomas. Cancer Genet Cytogenet 153: 64-68, 2004.

24. Nafe R, Glienke W, Burgemeister R, et al: Regional heterogeneity of EGFR gene amplification and nuclear morphology in glioblastomas. Analyt Quant Cytol Histol 26: 65-76, 2004. 